\title{
Affective Feedback in a Virtual Reality based Intelligent Supermarket
}

\section{Deba Pratim Saha}

Institute for Creativity, Arts and

Technology, Virginia Tech

Blacksburg, USA

dpsaha@vt.edu

R. Benjamin Knapp

Institute for Creativity, Arts and

Technology, Virginia Tech

Blacksburg, USA

benknapp@vt.edu
Thomas L. Martin

Institute for Creativity, Arts and

Technology, Virginia Tech

Blacksburg, USA

tImartin@vt.edu

Permission to make digital or hard copies of part or all of this work for personal or classroom use is granted without fee provided that copies are not made or distributed for profit or commercial advantage and that copies bear this notice and the full citation on the first page. Copyrights for third-party components of this work must be honored. For all other uses, contact the owner/author(s).

Copyright held by the owner/author(s).

UbiComp/ISWC'17 Adjunct, September 11-15, 2017, Maui, HI, USA ACM 978-1-4503-5190-4/17/09.

https://doi.org/10.1145/3123024.3124426

\begin{abstract}
The probabilistic nature of the inferences in a context-aware intelligent environment (CAIE) renders them vulnerable to erroneous decisions resulting in wrong services. Learning to recognize a user's negative reactions to such wrong services will enable a CAIE to anticipate a service's appropriateness. We propose a framework for continuous measurement of physiology to infer a user's negative-emotions arising from receiving wrong services, thereby implementing an implicit-feedback loop in the CAIE system. To induce such negative-emotions, in this paper, we present a virtualreality (VR) based experimental platform while collecting real-time physiological data from ambulatory wearable sensors. Results from the electrodermal activity (EDA) data analysis reveal patterns that correlate with known features of negative-emotions, indicating the possibility to infer service appropriateness from user's reactions to a service, thereby closing an implicit-feedback loop for the CAIE.
\end{abstract}

\section{Author Keywords}

Physiological Computing; Affective Feedback

\section{ACM Classification Keywords}

1.5.4 [Pattern Recognition]: Applications; H.5.1 [Multimedia Information Systems]: Virtual Reality

\section{Introduction}

The widespread adoption of portable networked computing devices has pushed these systems away from the limited desktop scenario into the wild, ever closer to the activities of our daily lives. Mark Weiser's vision of "invisible, 


\section{Affect-aware CAIE}

A new branch of computing has emerged called Affective Computing (AC) that "relates to, arises from and deliberately influences human emotions" (Rosalind

Picard, MIT Media Lab).

$A C$ techniques enable us to infer a user's emotional valence (surrogates for likes or dislikes) from a variety of modalities such as physiological variables (more prominently electrodermal activity, heart-rate variability, respiration-rate and skintemperature) along with facial expressions, voice prosody, gesture and actions.

Affective interfaces and agents have been successfully shown to improve overall usability, quality of interaction of a system and sometimes even helping reduce user's experienced stress even when performing frustrating tasks, making the system more human-centric [18]. Human affect forms the implicit channel of communication and augments the meaning conveyed explicitly by language and actions [4]. everywhere computing"-technology that disappears from our cognitive fronts and lives in the woodwork everywhere [20]-is closer to fruition than ever before. In such a pervasive computing scenario, where a user often interacts with a context-aware computing infrastructure consisting of a dynamic set of devices with distributed focus of interaction [17], seamless delivery of a context-aware service is a challenge mainly due to the lack of a pervasive interface to ascertain a user's preferences. It would defeat the purpose of having an inference-based context-aware system to stop after providing an intelligent service and explicitly query the user about its appropriateness, in addition to introducing a cognitive load on the user. If, on the other hand, the pervasive computational system could implicitly infer a user's (dis)approval about the service's (in)appropriateness from their behavioral or physical cues, the need for stopping and asking the user could be completely eliminated. Implicitly learning a user's disapproval of an intelligent service in a multi-turn interaction, has been widely used in informationretrieval domain to model search relevance [21].

A CAIE may be limited by sensory data to infer a user's context, thus producing services that are not properly aligned with a user's current needs (sometimes even hindering their progress towards their goals), initiating a phenomenon called technostress in the user. Affective Computing (see sidebar) techniques provide feasible methods to infer such technostressed states from physiological signals establishing an implicit channel of communication between the interacting agents (the CAIE and the user), which improves the quality of interaction by attaining convergence in communication [4]. In a recent work [16], the authors have explored the idea of a physiology based implicit affective-feedback loop in a CAIE to recognize these technostressed states induced by the inappropriate services from the CAIE, by mocking up a warehouse scenario in their laboratory.

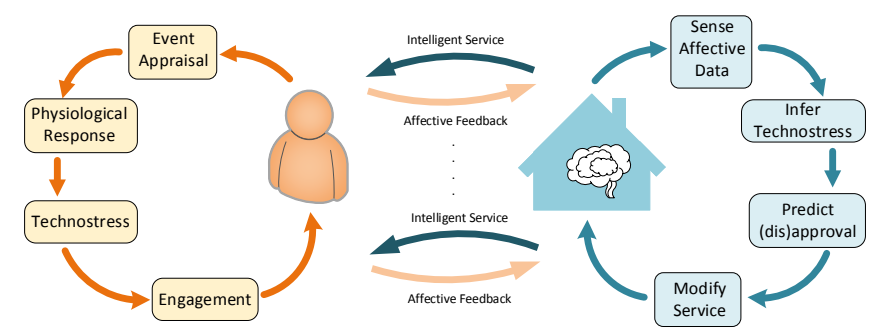

Figure 1: Interaction-process Model of Service Delivery and Affect Generation in an Intelligent Environment with an Affective Feedback.

In order to test our hypothesis of implementing an implicitfeedback loop based on validated physiological signal patterns, we present our experimental setup of a VR based supermarket with navigation-assist, wherein a user navigates the virtual supermarket in a fully position tracked space, while wearing an Empatica E4 wristband continuously acquiring real-time physiological data [8]. With the current setup, we intend to improve upon prior work such as [16] where the authors mocked-up a warehouse scenario in their lab having limited immersive experience for the participants. This current platform enables us to immerse a participant in a realistic CAIE, while taking a flexible, repeatable and systematic approach in evaluating in the interaction model shown in Figure 1 and Figure 2.

\section{Background}

A CAIE is a space where a pervasive intelligent system senses a user's current activities and goals, while consistently maintaining a coherent interaction across multiple devices [17]. Unlike a typical computing user interface (UI) (with traditional input-output devices), UI design for CAIE often poses a challenging problem for the designer due to the pervasiveness of the computing infrastructure and the lack of a single continuous medium of interaction, motivating the need to rethink the interface design [17]. 


\section{Technostress in CAIE}

Technostress is the perception of hassles due to system response delay during interaction with technology [14], which produces elevated levels of stress hormones (e.g., cortisol and adrenaline) and heightened activity of the sympathetic division of the autonomic nervous system (ANS), which regulates heart-rate variability (HRV), electrodermal activity (EDA) skin temperature (ST) and blood pressure (BP) [14].

Technostress has a physiological signature similar to that produced during a psycho-social stress [9, 14], which is a well studied field under AC [12, 22]. In her seminal work, Kreibig points out that ANS responses appear more pronounced in negative emotions (such as technostress, anger) compared to positive emotions (such as happy, excited) [9].

A leading cause of technostress is "achievement stress" which is heightened in system failures during time-pressured tasks, i.e., tasks having hard-deadlines associated with them.

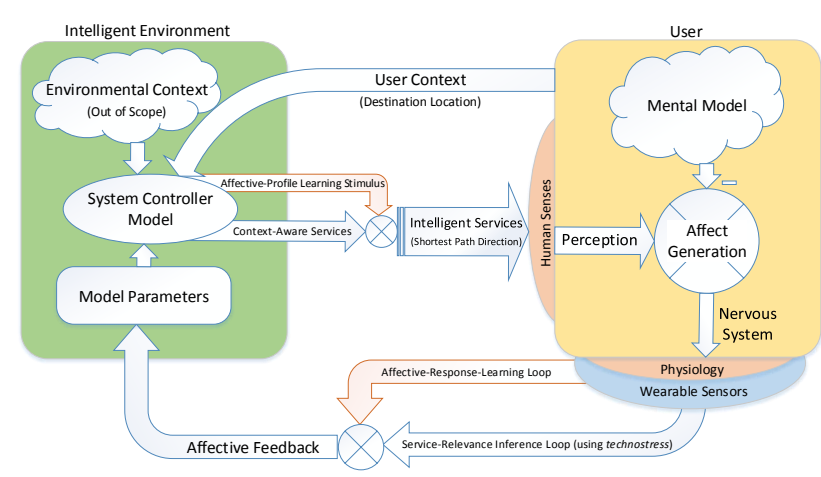

Figure 2: Schematic of an Affective Feedback in an CAIE with a Physiological Response Learning Framework (Modified from [16])

Shafer et al. note that a self-learning adaptive interface is well suited for a pervasive CAIE system, however, it should provide an immediate undo mechanism as a remedy for inappropriate services [17]. We argue, AC techniques to recognize user's reactions (such as, technostress - see sidebar) to inapproriate services can be used to provision for this undo mechanism, as explored in [16].

AC systems that map visual, auditory, behavioral or physiological cues to affective states based on patterns of change as seen in a ground truth datasets are termed as Affective feedback (AF) loops [11]. AF loops have been effectively implemented in reorienting smart-home behavior by inferring user (dis)approval using vision based facial feature understanding [2]. However, we argue that a vision based facial expression recognition system limits the usability of the system due to sensing limitations. We posit that on-body wearable sensing of physiological signals alleviates the need for a centralized focal point of interaction, compared to a vision or audio based interfaces. In addition, physiological signals are potent signal streams for effectively sensing changes in ANS activity in response to technostressors [9].
Thus, continuous measurement of physiological signals to infer technostress for designing an implicit AF loop, as also explored in [16], is well suited for designing such AF loops in a CAIE and provides a novel opportunity to create Shafer's undo mechanism as previously alluded to.

\section{Interaction Model}

A direct outcome of our discussion so far is the schematic of a CAIE which periodically cycles between (a) providing intelligent services by recognizing user's context and (b) building and refining individualized behavioral model of a user's affective profile (using, say, physiological response patterns). This schematic is shown in Figure 2 which operates by a CAIE collecting sensory data to detect various aspects of user's context such as environmental (e.g., user identity, time, and location), physical (e.g., current activity and goals set by the user) and social (e.g., other users nearby, their relation with current user). Based on its inference regarding a user's context, the CAIE presents a service to assist them in their current goals. The appropriateness of this service vis-a-vis their goals is appraised by the user and a physiological response is generated through ANS activation (i.e. technostress or lack thereof). From the CAIE perspective, detecting technostress in a certain window following a service, using the physiological signals would imply user's (dis)approval of the service, thus prompting it to modify the service in the next iterations. This iterative nature of interaction is shown in the interaction model in Figure 1, which also opens up the possibility of an adaptive-window based multi-turn interaction where on identifying the buildup of technostress, the CAIE may ask clarifying questions, as shown in a recent work [10].

Need for Affective Profile Learning Framework

Collecting ground truth data for physiological signals from natural settings is difficult. In addition, they have some inherent challenges such as between-person variations 


\section{Paced Stroop Test (PST)}

\section{The Stroop color-word inter-} ference test demands that the user chooses the font color of a word which is depicting the name of either the same color as the font's color or a different color. In the congruent (C-PST) version of the test, the font color of the word and the name of the color depicted by the word match, whereas in the incongruent (IC-PST) version, they do not.

\section{A modified version of} this test is called Paced Stroop (code - https: //github.com/debapratimsaha/ PacedStroopTest), where each iteration of the Stroop test is programmed to be active for a stipulated time, say 3 seconds [22]. This task-pacing during the Stroop test has been shown to enhance the stress-inducing capability of Stroop test as compared to self-paced Stroop test, due to the need to expend increased amount of mental/cognitive effort in producing the correct response [13]. arising from personality differences [6] and biological predispositions [7]. Plarre et al. [12] argue that physiological response data from users while they are experiencing validated stressors such as mental arithmetic, public speaking etc. can be used as a practical method to collect annotated ground truth data. The Paced Stroop Test (see sidebar) is a validated laboratory stressor that has been widely used to annotate stress data [16, 22]. In [15], the authors have proposed to use physiological responses to music to learn an individual affective profile. Periodic presentation of such validated stimuli, collection of physiological data and refinement of userwise behavioral model from this data constitutes the physiological profile learning loop (see Figure 2).

\section{Experiment Design and Setup}

Supermarkets are potentially a good candidate to model as a CAIE, wherein Wahlster et al. [19] envision various intelligent interactions such as RFID tagged objects and ubiquitous web-connected shopping lists. One such novel service may be a simple navigation-assist inside the supermarket which finds the shortest direct path to cover the items on a dynamically changing shopping list (say, being updated in real-time by a partner). There has been recent commercial interest such as an in-store navigation-assist system introduced by Lowe's Supermarkets, and a smart shopping experience requiring no checkout designed by Amazon Inc.
Intelligent Supermarket in VR Experiment

In our prototype system, the navigation-assist will always try to find the shortest direct path from current location to the destination obtained from a grocery list. However, due to the dynamic nature of the list, as well as real-life issues such as latency in updating the list due to poor connectivity, the system may not always come up with an optimum path. Since the navigation-assist service is intended to help the customer achieve their goals faster, the wrong services (i.e. winding path) may cause achievement stress which is a potent cause of technostress. To design the instrumented supermarket as a CAIE, we have modeled an immersive VR based grocery store in a fully position tracked space at The Cube, Virginia Tech.

In our model, items are placed on shelves marked with serial numbers. Participants were informed that their shopping list was pre-populated with 10 items, and item numbers corresponding to the next item will be shown as an overlay on the supermarket scene in their VR-glasses (shown in Figure 3d). This unseen item-list gives a perception of a dynamically changing shopping list. Participants were informed that the system will highlight a direct path from current position to destination using horizontal green patharrows, while a vertical red-arrow will indicate the final destination visible from their current location (shown in Fig-

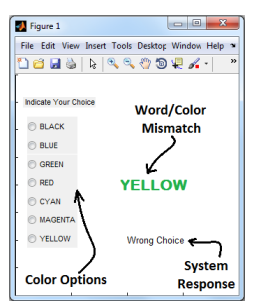

(a) PST Setup

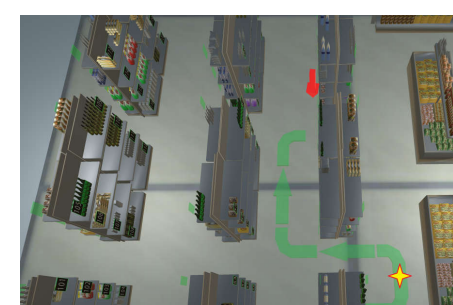

(b) Direct Path Top View

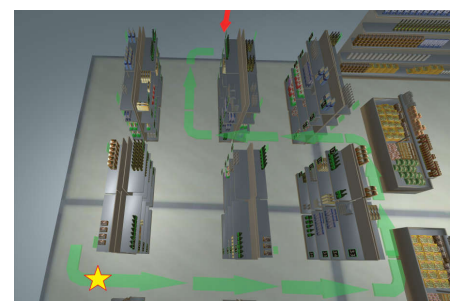

(c) Winding Path Top View

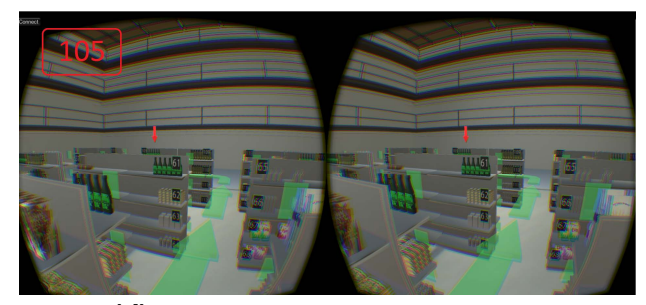

(d) Snapshot of a Virtual-Reality View 


\section{Hardware Setup}

Our setup consists of a Qualisys Motion Capture system with 24 Oqus5+ cameras for tracking reflective marker based rigid-bodies and an Oculus Rift DK2 as our VR glass. The Oculus Rift DK2 is connected to a laptop (say, Oculus Computer (OC) which is running the supermarket model in Unity, and is being carried by the participant in a backpack. For performing the experiment in Wizard-of-Oz fashion, the experimenter has a mirrored view of the participant's VR view (running on $\mathrm{OC}$ ), onto a local computer, say Experimenter Computer (EC) communicating over the local wireless network. Physiological data is collected using Empatica E4 wristband which streams time-stamped biosignal datastreams over Bluetooth to OC. The hotkey presses (VR event onsets) are time-stamped with the OC machine-time along with Empatica E4 data packets using custom code (available at https: //github.com/debapratimsaha/ EmpaticaUnityBLEClient). ure $3 d$ ). This red-arrow is essential for the user to create a mental model of the smart-service (i.e. a direct path), violating which may impart technostress. In reality, the experiment was conducted in a Wizard-of-Oz fashion, where-in the experimenter would listen to the participant speaking the next item number on their VR screen and activate the next path arrow by pressing a hotkey in the experimenter's view (see sidebar for the hardware setup). Some of the paths were an obvious direct path (see the top-view in Figure $3 b$ ) while some paths were deliberately made winding (see the top-view in Figure 3c) to create an impression of a system failure thus imparting technostress. Participants were asked to always follow the path indicated by green arrows, even if it is not a direct path. Out of the 10 items on the list, we provided correct service (CS: direct path) and wrong service (WS: winding path) for 5 items each.

\section{Paced Stroop Test (PST) Experiment}

For our experimental setup, we have used task pacing time of 3 seconds between each Stroop figure (shown in Figure $3 \mathrm{a}$ ), running for a total of 180 seconds, where one block of 60 seconds (i.e., 20 pairs) of C-PST is preceded and succeeded by 60 seconds each of IC-PST (i.e., $2 \times 20$ pairs). Physiological data was collected for the duration of 180 seconds. Although out of scope of this paper, analysis of this PST dataset may enable us to learn a user-specific response profile (discussed in Interaction Model section).

\section{Methods and Analysis}

Electrodermal Activity (EDA) Analysis

EDA is a reliable indicator of activation of the sympathetic division of ANS (SNS), which shows heightened activity during the experience of stress [7,9]. It is arguably the only physiological system that is activated solely by SNS, uncontaminated by the parasympathetic division (PSNS), making it a well established marker for SNS activity [16]. EDA signal is composed of a slow varying tonic and a rapidly changing phasic components. For EDA decomposition, we have used a deconvolution based method (see Ledalab [1]), wherein the measured EDA is deconvolved with an impulse response function (IRF) waveform to obtain the underlying compact sudomotor nerve-activity (SMNA) pulses. The IRF is modeled as a biexponential Bateman function $f(t)=\exp ^{\frac{-t}{\tau_{1}}}-\exp ^{\frac{-t}{\tau_{2}}}$ that explains the physiological processes of EDA generation [1], refer to Figure 4 for details.

Integrated Phasic Response (IPR) Analysis

Ledalab ${ }^{1}$ can decompose superposed EDA peaks into independent SMNA pulses, thus enabling the separation of phasic peaks. An advantage of Ledalab is the resulting phasic EDA has a zero baseline, enabling us to compute the time-integral of phasic EDA over a response window, which is a measure of sympathetic activation from the stimulus [1]. After decomposition, we slice individual SMNA peaks and reconvolve them with the IRF to obtain individual non-overlapping zero-baseline phasic EDA peaks. We take time-integral of these peaks, to obtain an EDA scoring measure defined as integrated phasic response (IPR) [1].

\section{EDA Analysis Results}

Our goal for this experiment was to identify instances of technostress due to wrong-services from a CAIE based on validated physiological indicators. We conducted a user study and have collected data from 7 participants (6 males, 1 female) under a research protocol approved by Virginia Tech (IRB-15-1193). Participants represented a wide range of nationality and ethnicity. The results from our batch analysis of EDA features, accumulated per event type show heightened sympathetic activation during WS events when compared to CS events based on validated physiological indicators. The results of this analysis will enable the CAIE

\footnotetext{
${ }^{1}$ Code available: https://github.com/brennon/Pypsy
} 


\begin{tabular}{ccc}
\hline User & CS & WS \\
\hline A & 523.15 & $\mathbf{5 5 5 . 4 3}$ \\
B & 235.7 & $\mathbf{3 6 3 . 2 1}$ \\
C & 80.6 & $\mathbf{1 5 1 . 8 5}$ \\
D & 51.41 & $\mathbf{7 8 . 8 7}$ \\
E & 7.77 & $\mathbf{5 2 . 7}$ \\
F & 14.54 & $\mathbf{8 8 . 3 3}$ \\
G & 67.65 & $\mathbf{8 6 . 2 9}$ \\
\hline
\end{tabular}

(a) Userwise IPR in Service Groups

\begin{tabular}{ccc}
\hline User & CS & WS \\
\hline A & $\mathbf{6 6}$ & 63 \\
B & 21 & $\mathbf{3 6}$ \\
C & 10 & $\mathbf{3 0}$ \\
D & 14 & $\mathbf{2 1}$ \\
E & 17 & $\mathbf{3 3}$ \\
F & 16 & $\mathbf{3 7}$ \\
G & 22 & $\mathbf{4 1}$ \\
\hline
\end{tabular}

(b) Userwise Number of Phasic peaks

\begin{tabular}{ccc}
\hline User & CS & WS \\
\hline A & 39.16 & $\mathbf{4 4 . 3}$ \\
B & $\mathbf{5 5 . 9 1}$ & 50.06 \\
C & 23.88 & $\mathbf{2 5 . 5 2}$ \\
D & 16.38 & $\mathbf{2 0 . 2 4}$ \\
E & 1.69 & $\mathbf{8 . 8}$ \\
F & 3.34 & $\mathbf{1 1 . 6 8 8}$ \\
G & $\mathbf{1 5 . 3 2}$ & 11.39 \\
\hline
\end{tabular}

(c) IPR per Peak in Service Group

Table 1: Userwise integrated phasic response (IPR) (in $\mu S s$ units) and Peak Count Analysis. Higher scores indicating stronger sympathetic activation, in each Correct Service

(CS)/Wrong Service (WS) pair for each user are bold-faced. to decide, when to ask clarifying questions in an adaptivewindow based multi-turn interaction as discussed in Interaction Model section.

The number of significant phasic peaks and time-integral of phasic peaks are widely used EDA features, wherein a higher number represents stronger sympathetic activation [5]. To perform the IPR analysis, the individual phasic peaks are thresholded to above $5 \%$ of the userwise maximum peak-amplitude to mark the significant peaks. Time-integral of these individual phasic peaks, where time is measured in seconds and phasic EDA in $\mu S$, are computed and accumulated for each type of services i.e. correct (CS) and wrong (WS) within their respective windows to obtain the IPR values (in $\mu S s$ units). The results are compiled in Table 1 where the bold-faced numbers are higher among the CS/WS pairs for each user. We can see that for all users, IPR during the WS events is higher than that during the CS events. In addition, IPR per Peak is computed by dividing the total IPR by the number of peaks following a service, then accumulating for each service type. We see that for five users, the IPR per Peak is higher during the WS events. The number of significant phasic EDA peaks is also compiled, and barring User A, we obtain higher number of significant phasic peaks during WS compared to CS events. Time-spans for each events depend on the length of the paths, however, WS events induce higher number of phasic peaks each with greater IPR (as seen in Table 1b-1c) indicating stronger SNS activation. It must be noted, that with a more liberal thresholding (say, 15\%) for peak significance, the results for User A in Table $1 \mathrm{~b}$ and for User B and $\mathrm{G}$ in Table 1c are consistent with the overall results.

Although, there are some users (esp. B and G) for whom the physiological indicators did not reflect these patterns, we have learned that such differences may arise from factors such as personality [6]. We do not have personality re-

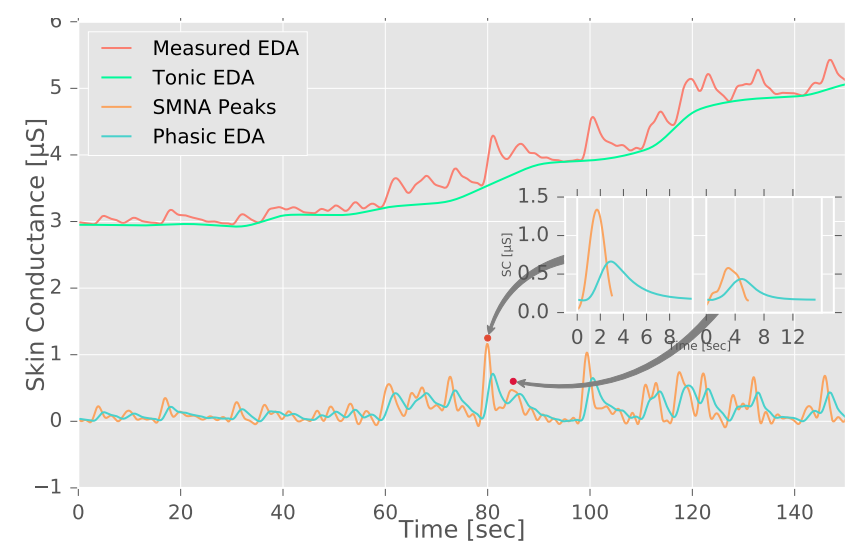

Figure 4: EDA Decomposition using Ledalab. Observe the tonic EDA follows the measured EDA signal, while sliced individual SMNA peaks are convolved with IRF to obtain zero-baselined phasic EDA (see inset). Notice the overlapped phasic peaks are separated as individual peaks.

lated data in our current dataset, however, we intend to add such qualitative data collection methods for our forthcoming phases of the experiment.

\section{Discussion}

With this current work, we sought to identify user-independent physiological indicators of stress experienced by users in CAIE, when they receive an inappropriate service. Table 1 shows that the number of phasic EDA peaks and average IPR in these peaks is higher during WS events, i.e. more numbers of larger phasic EDA peaks are produced during WS events. From this, we can infer that users show higher sympathetic activation during the WS events compared to the CS events. Thus, from our experimental dataset in a VR environment, we observed patterns in EDA signal across users during such WS (i.e. inappropriate or wrong services), that have been shown to be correlated with negative emotional states [9] such as frustration. The hypothesis behind our interaction framework as discussed in Interaction 


\section{Conclusion}

In this paper, we have proposed a novel system architecture to employ affective computing techniques to identify a user's technostressed states arising from wrong services received from an intelligent environment. Successful identification of technostressed states following a service, which would imply it's inappropriateness, can be used as a feedback signal in order to refine the services in subsequent turns. To evaluate this hypothesis, we have designed a controlled experimental platform in a VR setup providing intelligent services, and occasionally providing wrong services, while collecting real-time physiological data. The results from EDA signal analysis from our study conducted in the experimental platform show heightened sympathetic activation during wrong services, indicating onset of negative-emotional states such as technostress. These results are encouraging as we continue to refine our setup and analysis.
Model section, rests on the successful identification of such affective states from physiological data. Our results from the batch analysis show greater number of phasic EDA peaks each having higher average IPR, both of which are independent evidences of stronger sympathetic activation in users while experiencing technostress in a CAIE. Although individual eventwise analysis is not conclusively consistent across all users, however, with further analysis of more EDA features and HRV signals, we hope to improve upon the granularity of these discriminatory inferences to, possibly, a single window following each service. Nevertheless, the patterns from this group analysis will enable a CAIE to improve a multi-turn interaction (see Interaction Model section) using features of technostress.

In addition to continued analysis of the physiological signals, we are also refining our experimental protocol, in order to gain more insights into known influencers of human affective responses such as their personality [6], thereby helping us improve our inferences. For instance, a recent work has demonstrated that the daily usage pattern of a mobile phone is predictive of a person's personality types [3]. While collecting such mobile usage data is out of the scope of our work, we intend to add qualitative data collection methods such as personality questionnaire.

\section{Acknowledgment}

The authors would like to thank Institute for Creativity, Arts and Technology at Virginia Tech for supporting this project, especially Reza Tasooji and Zachary Duer for helping us model the $3 \mathrm{D}$ environment.

\section{REFERENCES}

1. Mathias Benedek and Christian Kaernbach. 2010. A Continuous Measure of Phasic Electrodermal Activity. Journal of Neuroscience Methods 190, 1 (June 2010), 80-91.
2. Kuderna-Iulian Benţa, Cremene Marcel, and Todica Valeriu. 2009. Towards an Affective Aware Home. In Ambient Assistive Health and Wellness Management in the Heart of the City. Number 5597 in Lecture Notes in Computer Science. Springer Berlin Heidelberg, 74-81.

3. Gokul Chittaranjan, Jan Blom, and Daniel Gatica-perez. 2011. Who's Who with Big-Five: Analyzing and Classifying Personality Traits with Smartphones. In In Proc. Int'l Symposium on Wearable Computers (ISWC).

4. R. Cowie, E. Douglas-Cowie, N. Tsapatsoulis, G. Votsis, S. Kollias, W. Fellenz, and J.G. Taylor. 2001. Emotion Recognition in Human-Computer Interaction. IEEE Signal Processing Magazine 18, 1 (Jan. 2001), 32-80.

5. Benjamin Ultan Cowley and Jari Torniainen. 2016. A Short Review and Primer on Electrodermal Activity in Human Computer Interaction Applications. arXiv:1608.06986 [cs] (Aug. 2016).

6. Andrew Crider. 2008. Personality and Electrodermal Response Lability: An Interpretation. Applied Psychophysiology and Biofeedback 33, 3 (May 2008), 141-148.

7. George S. Everly and Jeffrey M. Lating. 2013. The Anatomy and Physiology of the Human Stress Response. In A Clinical Guide to the Treatment of the Human Stress Response. Springer New York, 17-51.

8. M. Garbarino, M. Lai, D. Bender, R.W. Picard, and S. Tognetti. 2014. Empatica E3 - A wearable wireless multi-sensor device for real-time computerized biofeedback and data acquisition. In 2014 EAl 4th International Conference on Wireless Mobile Communication and Healthcare (Mobihealth). 39-42. 
9. Sylvia D. Kreibig. 2010. Autonomic Nervous System Activity in Emotion: A Review. Biological Psychology 84, 3 (July 2010), 394-421.

10. Sarah Mennicken, Oliver Zihler, Frida Juldaschewa, Veronika Molnar, David Aggeler, and Elaine May Huang. 2016. "It's Like Living with a Friendly Stranger": Perceptions of Personality Traits in a Smart Home. In Proceedings of the 2016 ACM International Joint Conference on Pervasive and Ubiquitous Computing (UbiComp '16). ACM, 120-131.

11. Domen Novak, Aniket Nagle, and Robert Riener. 2014. Linking Recognition Accuracy and User Experience in an Affective Feedback Loop. IEEE Transactions on Affective Computing (2014), 168-172.

12. K. Plarre, A. Raij, S.M. Hossain, A.A. Ali, M. Nakajima, M. al'Absi, E. Ertin, T. Kamarck, S. Kumar, M. Scott, Daniel Siewiorek, A. Smailagic, and L.E. Wittmers. 2011. Continuous Inference of Psychological Stress from Sensory Measurements Collected in the Natural Environment. In 2011 10th International Conference on Information Processing in Sensor Networks. 97-108.

13. Patrice Renaud and Jean-Pierre Blondin. 1997. The Stress of Stroop Performance: Physiological and Emotional Responses to Color-word Interference, Task Pacing, and Pacing Speed. International Journal of Psychophysiology 27, 2 (Sept. 1997), 87-97.

14. Rene Riedl, Harald Kindermann, Andreas Auinger, and Andrija Javor. 2013. Computer Breakdown as a Stress Factor during Task Completion under Time Pressure: Identifying Gender Differences Based on Skin Conductance. Advances in Human-Computer Interaction 2013 (Oct. 2013).

15. Deba Pratim Saha, Brennon Bortz, Wei Huang, Thomas Martin, and Benjamin Knapp. 2016. Affect-Aware Intelligent Environment Using Musical
Cues as an Emotion Learning Framework. In 2016 12th International Conference on Intelligent Environments (IE). 178-181.

16. Deba Pratim Saha, Thomas L. Martin, and R.Benjamin Knapp. 2015. Towards Incorporating Affective

Feedback into Context-Aware Intelligent Environments. In 2015 International Conference on Affective Computing and Intelligent Interaction (ACII). 49-55.

17. Steven A. N. Shafer, Barry Brumitt, and J. J. Cadiz. 2001. Interaction Issues in Context-Aware Intelligent Environments. Hum.-Comput. Interact. 16, 2 (Dec. 2001), 363-378.

18. Nik Thompson and Tonya McGill. 2015. Affective Human-Computer Interaction. In Encyclopedia of Information Science and Technology, 3e. 3712-3720.

19. Wolfgang Wahlster, Michael Feld, Patrick Gebhard, Dominikus Heckmann, Ralf Jung, Michael Kruppa, Michael Schmitz, Lübomira Spassova, and Rainer Wasinger. 2010. The Shopping Experience of Tomorrow: Human-Centered and Resource-Adaptive. In Resource-Adaptive Cognitive Processes. Springer Berlin Heidelberg, 205-237.

20. Mark Weiser. 1991. The Computer for the 21st Century. Scientific American 265, 3 (Sept. 1991), 94-104.

21. Ryen W. White. 2016. Interactions with Search Systems. Cambridge University Press.

22. J. Zhai and A. Barreto. 2006. Stress Detection in Computer Users Based on Digital Signal Processing of Noninvasive Physiological Variables. In 28th Annual International Conference of the IEEE Engineering in Medicine and Biology Society, 2006. EMBS '06. 1355-1358. 